(1968). The data indicate that the visual magnitude of 3C 273 was rather erratic with a total variation of about 0.1 magnitude.

Observations of 3C 273 for the years 1968 (Burkhead and Parvey 1968), 1969 (Burkhead 1969), and 1970 (this note) are presented in Figure 1. The errors of the observations can be estimated by the standard deviations, $\boldsymbol{\sigma}$, of the comparison stars from their accepted photoelectric values. These standard deviations are

$$
\sigma_{v}= \pm 0.017, \sigma_{B-V}= \pm 0.028, \sigma_{U-B}= \pm 0.04 \text {. }
$$

It should be noted that the $(U-B)$ color indices observed at McDonald Observatory $(1969,1970)$ are more negative than those observed in Bloomington. The hour angle of the 3C 273 observations at McDonald was large and there may be some difficulty in the reduction techniques. However, $(U-B)$ color indices equaling those obtained at McDonald have been measured locally. It is therefore believed that these color variations are real.

\title{
REFERENCES
}

Burkhead, M. S. 1969, Pub. A.S.P. 81, 691.

Burkhead, M. S., and Parvey, M. I. 1968, Pub. A.S.P. 80, 483.

\section{THE NATURE OF BD $+17^{\circ} 4708$}

JUDITH G. COHEN AND JESSE L. GREENSTEIN

Hale Observatories

Carnegie Institution of Washington

California Institute of Technology

Received June 4, 1970

Oke, Greenstein, and Gunn (1966) called the G0 star BD $+17^{\circ} 4708$ a field horizontal-branch star. They determined its effective temperature to be $6000^{\circ} \mathrm{K}$, and its surface gravity as $\log g=3.0$. In his study of field horizontal-branch stars, Newell (1969) found $+17^{\circ} 4708$ to be the reddest such star, and it occupied a critical position in his plot of $\boldsymbol{\theta}_{e}$ vs. $\log g$, serving to separate more clearly the groups he calls disk horizontal-branch and halo horizontalbranch stars. It is the purpose of this note to indicate that $+17^{\circ} 4708$ is a G subdwarf, possibly slightly evolved, rather than a field horizontal-branch star. 
The effective temperature of $+17^{\circ} 4708$ was determined by two independent methods, one involving the profile of $\mathrm{H} \gamma$ and the other the slope of the continuum. Two calibrated spectra were available taken with the 200-inch Hale telescope on baked IIa-O plates with a dispersion of $9.0 \AA \mathrm{mm}^{-1}$. The $\mathrm{H} \gamma$ profiles were measured for each wing of the line on each plate and then averaged, to give the profile shown in Figure 1. Also shown in Figure 1 is an $\mathrm{H} \gamma$ profile from a radiative equilibrium, LTE model obtained from computer programs written by Dr. S. E. Strom, Mr. R. L. Kurucz, and Dr. D. M. Peterson. It is clear that the $\mathrm{H} \gamma$ profile for the model with $T_{\text {eff }}=6250^{\circ} \mathrm{K}, \log g=4.0$, and $\mathrm{Z} / \mathrm{Z}_{\odot}=0.1$ satisfactorily matches the observed stellar profile.

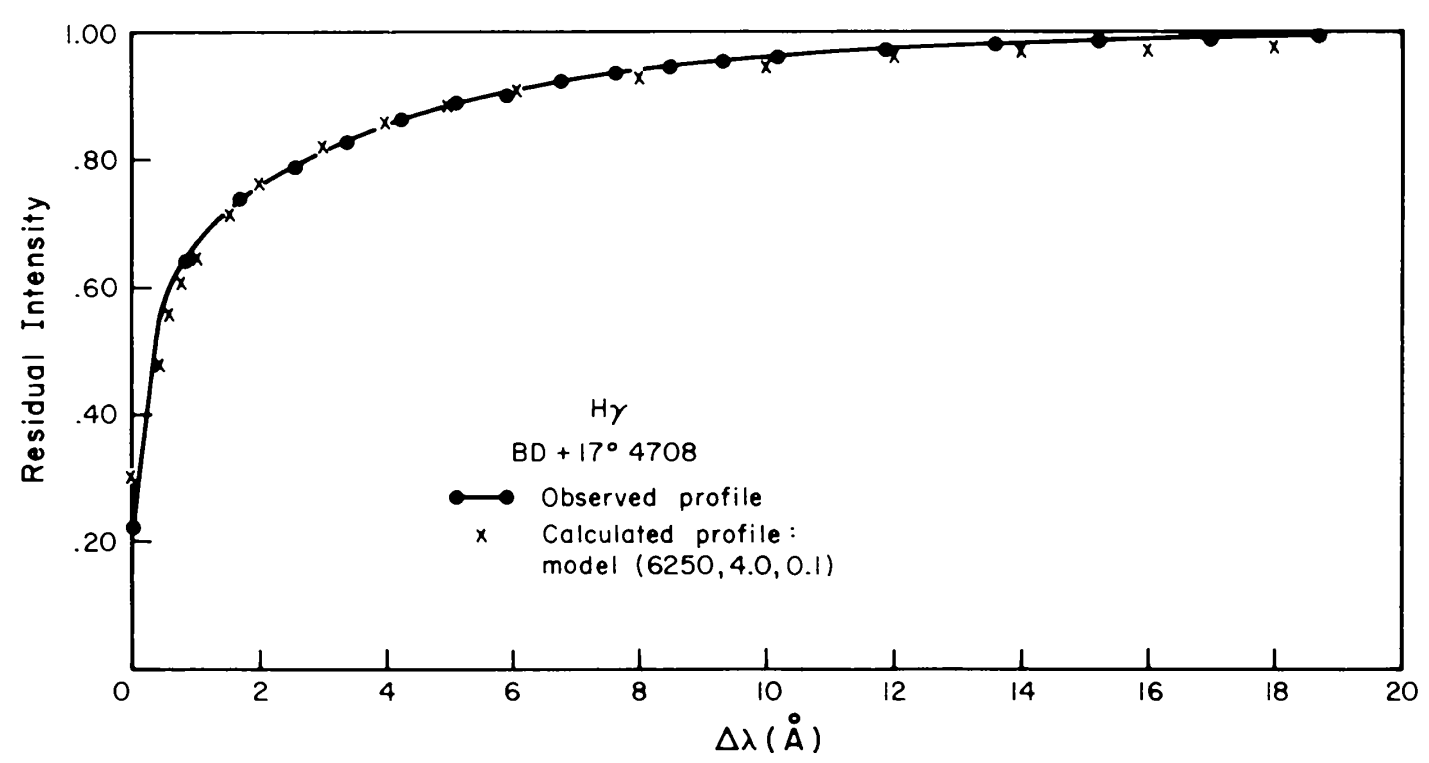

FIG. 1 - Mean, reflected $\mathrm{H} \gamma$ profile in $\mathrm{BD}+17^{\circ} 4708$. Circles represent observed profile; crosses, calculated profile at $T_{\text {eff }}=6250^{\circ} \mathrm{K}, \log g=4.0$, and one-tenth solar metal abundance.

Several spectrophotometric scans of the star were kindly made available by Dr. J. B. Oke, and an average of these is shown in Figure 2, where we have used the absolute calibration determined by Oke and Schild (1970). Because the star is only moderately metal deficient, it is necessary to correct the observed scan for the effects of "ine blanketing. This had not been done by Oke, Greenstein, and Gunn (1966). Therefore the fraction of the flux removed by the lines of the appropriate wavelength ranges was measured on 


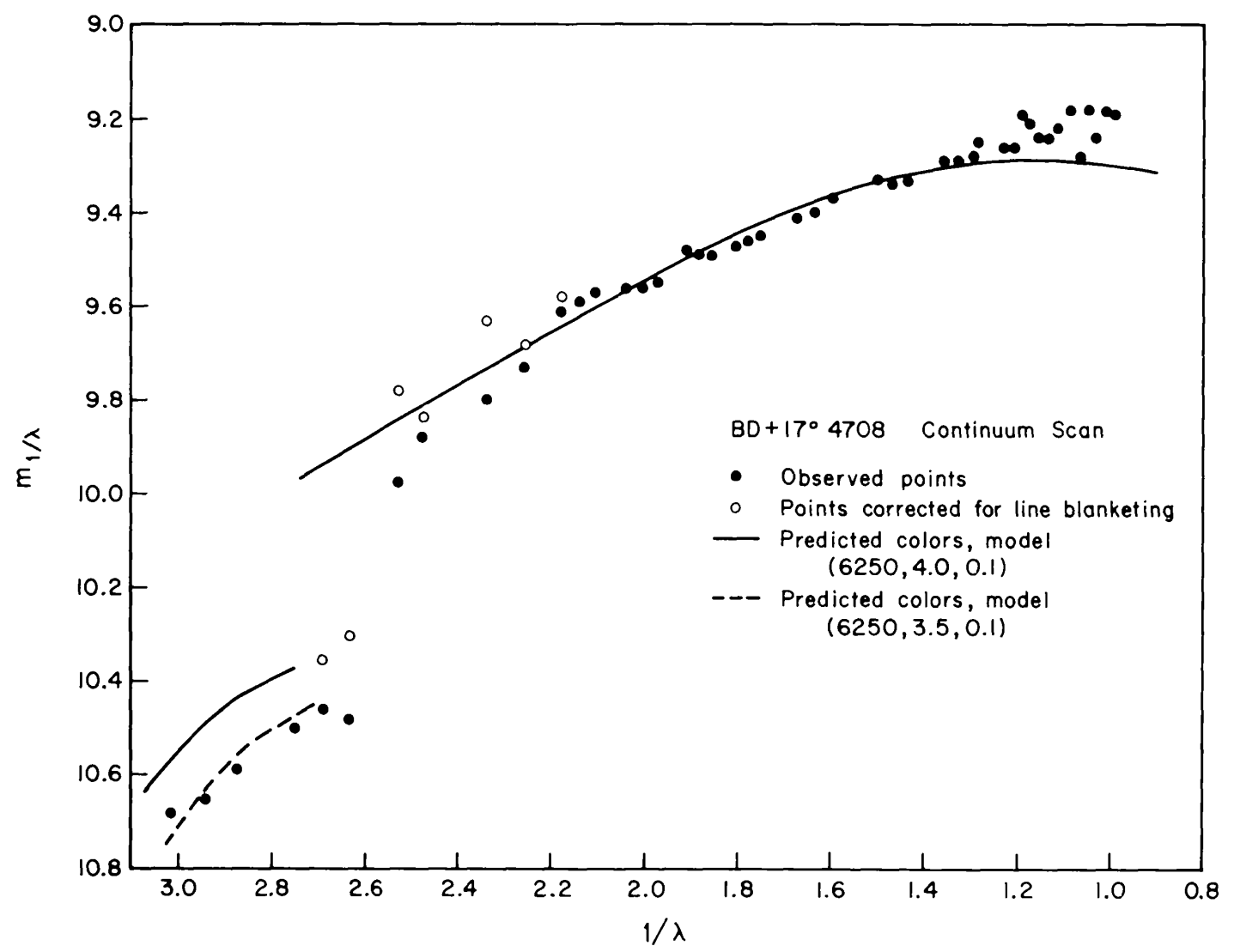

FIG. 2-Spectrophotometric scans of continuum by Dr. J. B. Oke. Observed points (filled circles) are corrected for blanketing and plotted as open circles. The solid line is the predicted flux for the model with $\log g=4.0$; the dashed line that for $\log g=3.5$. The points below the Balmer limit require the higher surface gravity.

the two plates, and the observed scan was corrected as shown in Figure 2. The effective temperature determined from the scan, $T_{\text {eff }}=6250^{\circ} \mathrm{K}$, agrees with that from the $\mathrm{H} \gamma$ profile, and hence there is little interstellar reddening. The inclusion of line-blanketing corrections forces one to conclude that the surface gravity must be greater than $\log g=3.0$, and is probably about $\log g=4.0$. Thus the star is perhaps slightly evolved off the main sequence, which has a $\log g$ of 4.3 at this temperature. Unfortunately, our plates do not go below $3700 \AA$, so that we cannot correct the ultraviolet fluxes. Because the continuum is heavily obscured by lines, including the Balmer series, below $4000 \AA$ the first few blanketing corrections are too small. The excess flux in the red is probably due to $l$ ackwarming. A similar effect in the red was observed with broad-band photometry by Johnson, MacArthur, and Mitchell (1968). 
A second method of determining the surface gravity is through the use of the ionization equilibrium of iron. Equivalent widths of approximately 75 lines were measured and abundances were obtained using the method described in Cohen (1968). However, recent laboratory measurements of the $g f$ values for Fe I by Garz and Kock (1969) have revealed not only a change in the absolute normalization from the values of Corliss and Warner (1964), but also an error which depends on the excitation potential of the upper level of the lines. We have so corrected all the Fe I $g f$ values, as a function of the excitation potential of the upper level. It is not yet clear whether a correction should be applied to the Fe II transition probabilities, and hence we cannot use the ionization equilibrium of iron to accurately deduce the surface gravity. We find, however, that the observed equivalent widths yield abundances of $\mathrm{Cr}_{\mathrm{I}}$ and $\mathrm{Cr}$ II, of $\mathrm{Ti}_{\mathrm{I}}$ and $\mathrm{Ti}$ II, and of $\mathrm{Fe}_{\mathrm{I}}$ (using new gf values) and Fe II (using approximately scaled $g f$ values) which are within 0.2 in the log of each other, when the model $(6250,4.0$, 0.1 ) is used. The star has an abundance of iron a factor of 10 lower than that of the sun; calcium, titanium, and magnesium appear to be deficient by a somewhat smaller factor, as often occurs in metalpoor stars.

A further argument that $+17^{\circ} 4708$ is not a field horizontal-branch analog is based on its spatial velocities $U, V$, and $W$. The values given by Eggen (1964), assuming that the star is a G0 subdwarf with $M_{V}=4.6$, are $\left(+252,-270,+43 \mathrm{~km} \mathrm{sec}^{-1}\right)$ respectively. If the star were a field horizontal-branch star, it would have $M_{V}=0.5$ to 1.5 , and its space velocities would become incredibly large. One would then be surprised that the star is only moderately metal deficient, because of the high correlation of $\delta(U-B)$ with space motion.

In résumé, we have redetermined the atmospheric parameters of the star $\mathrm{BD}+17^{\circ} 4708$. Evidence from a scan corrected for line blanketing, from the line spectrum, and from kinematic considerations, leads us to conclude that the star is a slightly evolved G0 subdwarf, with a metal deficiency of approximately a factor of 10 , rather than a field analog of the horizontal-branch stars in globular clusters. 
We thank Professor Oke for the use of his scans, and for helpful discussion. We are grateful to Dr. Strom, Mr. Kurucz, and Dr. Peterson who wrote many of the computer programs used in this investigation. One of us (J.G.C.) gratefully acknowledges the support of a National Science Foundation traineeship.

\section{REFERENCES}

Cohen, J. G. 1968, Ap. J. 154, 179.

Corliss, C. M., and Warner, B. 1964, Ap. J. Suppl. 8, 395.

Eggen, O. J. 1964, Royal Obs. Bull No. 84.

Garz, T., and Kock, M. 1969, Astr. and Ap. 2, 277.

Johnson, H. L., MacArthur, J. W., and Mitchell, R. I. 1968, Ap. J. 152, 465.

Newell, E. B. 1969, Dissertation, Australian National University.

Oke, J. B., Greenstein, J. L., and Gunn, J. 1966, in Stellar Evolution, R. F. Stein and A. G. W. Cameron, eds. (New York: Plenum Press), p. 399.

Oke, J. B., and Schild, R. E. 1970, Ap. J. 161, 1015.

\section{NOTE ON THE LIGHT VARIATION OF GAMMA CORONAE BOREALIS}

R. TIPPETS AND S. K. WILCKEN

Department of Physics and Astronomy Brigham Young University

Received July 27, 1970

Percy (1970) has recently published some photometric observations of $\gamma$ Coronae Borealis indicating a light amplitude of $0^{\mathrm{m}} 05$ in yellow light in a period of approximately 0d03. Fernie (1969) had concluded earlier that the star may be a $\delta$ Scuti variable of short period on the basis of 16 scattered observations.

Observations of $\gamma \mathrm{CrB}$ made in yellow light, relative to $\theta \mathrm{CrB}$, were obtained on July 7 and July 12, 1970 with the 24-inch reflecting telescope of Brigham Young University. A refrigerated 1P21 photomultiplier and five ten-second integrations per observation were used. The differential magnitudes in the sense of $\theta \mathrm{CrB}$ minus $\gamma \mathrm{CrB}$ are displayed in Figure 1. Small differential magnitude corrections for atmospheric extinction have been applied to the data points. 